\title{
Spin glass state in high-temperature superconductors
}

\author{
V A CHERENKOV \\ All-Union Research Scientific Institute of Metrological Service, 117334 Moscow. \\ Andreevskaya nab.2. Moscow. USSR
}

Abstract. The glass state of high-temperature superconductors in the models of Josephson
weak links is studied. Reversibility phenomena in weak magnetic fields are outlined.

Keywords. Spin glass state; Josephson weak links.

\section{Introduction}

The metal-oxide superconductors, $\mathrm{La}-\mathrm{Ba}-\mathrm{Cu}-\mathrm{O}$ and $\mathrm{Y}-\mathrm{Ba}-\mathrm{Cu}-\mathrm{O}$, display superconducting glass properties analogous to those of spin glasses (Mullar et al 1987; Cai et al 1987; Ishizawa et al 1987). A percolation theory of weak Josephson link granular superconductors appears to reflect correctly the principal physical features of high- $T_{c}$ ceramic superconductors typical for a glass phase (Sajeev and Lubensky 1985; Ebner and Stroud 1985; Clem 1988; Klimento et al 1987). Here we discuss the behaviour of the isothermal $\chi^{\mathrm{ZFC}}$ and thermoresidual magnetization $\chi^{\mathrm{FC}}$, the anomalous large magnetic viscosity value. the continuous spectrum of relaxation time, the hysteresis effects, irreversibility phenomenon, nonergodicity (Clem 1988; Klimento et al 1987; Mazaki et al 1987). This work aims at determining the basic features of a glass phase and the estimation of maximum values of critical parameters in the Ising model with the transition temperature into the superconducting state and the upper critical field of high- $T_{c}$ superconducting ceramics in the model of weakly coupled weak Josephson link clusters.

\section{Weak Josephson interaction in superconducting contact models}

The existing models of Josephson junctions with weak interaction include planar $\mathrm{X}-\mathrm{Y}$ model of randomly coupled superconducting clusters, lattice model with randomly distributed Josephson junctions with the weak link affected by the external magnetic field (Sajeev and Lubensky 1985), the model of random orientation of superconducting loops in the external magnetic field (Ebner and Stroud 1985) and the model of lattice of superconducting filaments (Vinokur et al 1987). Of these only the last two permit analytical solution.

The model of random orientation of superconducting loops in the external magnetic field seems to be nearer to the real granular superconductors than the model of superconducting filament lattice. We however show that both models result in a superconducting state analogous to the weak Josephson link system corresponding to the Sherrington-Kirkpatrick spin glass model.

In the present model, the size of superconducting grains, filaments and pellets is smaller than the magnetic field penetration depth and the coherence length $\xi_{0}$ which 
provides the existence of screening supercurrents in magnetic fields $d \leqslant \lambda, \xi_{0}$. A large cluster possesses numerous superconducting states of equal energy and current. This ensures the principal state to be degenerate and nonergodic. The metastable character of states leads to a strong dependence of properties on the magnetic hysteresis of sample.

\subsection{Single superconducting loops model: Characteristic features of} the spin glass phase

Consider a superconducting cluster of $\mathrm{N}$ grains in a normal matrix. Let the grain centre be at $x_{i}$ and let the grains be weakly coupled. $\mathscr{J} N \lesssim k T$ with the Josephson junction.

$$
\begin{aligned}
& \hat{\mathscr{H}}=-\sum_{\langle i j\rangle} \mathscr{J}_{i j} \cos \left(\phi_{i} \dot{-} \phi_{j}-A_{i j}\right) \\
& A_{i j}=2 \pi / \phi_{0} \int_{i}^{j} \bar{A} \overline{d l}
\end{aligned}
$$

where $\mathscr{J}_{i j}$ is the Josephson constant, and in eq. (1) the integral is taken between grains $i$ and $j$. Assuming a canonical distribution for the magnetic moment $\mu$ and dc and ac magnetic susceptibilities $\chi_{\text {d.c. }}$ and $\chi_{\text {a.c. }}$, we have general expressions, see (Ebner and Stroud 1985)

$$
\begin{aligned}
& \mu=-[\partial F / \partial H]_{T} \\
& \chi_{\text {d.c. }}=[M / H]_{T} \\
& \chi_{\text {a.c. }}=[\partial M / \partial H]_{T}
\end{aligned}
$$

where $F$ is the free energy of the system, $M$ is the magnetization, $H$ is the external magnetic field, []$_{T}$ is the isothermal state at temperature $T$.

It is evident that the degeneracy of the principal state of the superconductor is determined from the phase factor in (1) providing a frustration (Sajeev and Lubensky 1985; Ebner and Stroud 1985). The principal state energy is quantized on the elementary magnetic flux quantum $\phi_{0}=h / 2 e$. It is easy to show that in the model of single superconducting loops, $\mathscr{F}_{i j} \equiv \mathscr{F}_{0}$. The principal state energy can be represented in the form

$$
\begin{aligned}
& \varepsilon=\varepsilon_{0}\left(\phi / \phi_{0}\right)^{2}\left[\left(\phi / \phi_{0}\right)-1\right] \\
& \varepsilon_{0}=2 \pi^{2} \mathscr{J} / N ; \phi=H S, 0 \leqslant H \leqslant \phi_{0} / S
\end{aligned}
$$

The function $\varepsilon(H)$ has analogous view in any interval

$$
\left(\phi_{0} / S\right)(n-1) \leqslant H \leqslant n \phi_{0} / S ; n \in\{N\} .
$$

DC magnetic susceptibility $\chi_{\text {d.c. }}$ corresponding to the thermodynamical equilibrium state of the superconductor should differ from that of $\chi_{\text {a.c. }}$, taking into account nonequilibrium properties of the matter. The difference between $\chi_{\text {d.c. }}$ and $\chi_{\text {a.c. }}$ is common for ceramic high- $T_{c}$ superconductors (Klimento et al 1987; Mazaki et al 1987). The same feature is characteristic for a spin glass also (Binder and Young 1986). From the Helmholtz energy standard expressions for magnetic moment and configurational 
mean []$_{c}$, see (Ebner and Stroud 1985) we obtain

$$
\chi_{\text {d.c. }}=\left[[(\partial \varepsilon / \partial H) / H]_{T}\right]_{c} ; \chi_{\text {a.c. }}=\left[\partial / \partial H[\partial \varepsilon / \hat{c} H]_{T}\right]_{c}
$$

For the assembly of identical single superconducting loops (SSL), the result is

$$
\chi_{\text {a.c. }}=\mathscr{J} n S^{2} / \phi_{0}: n=\left(2 \pi^{2} / N\right)^{3}
$$

at $\quad \partial^{2} \phi / \partial H^{2} \ll(\delta \phi / \partial H)^{2}$.

In the general case

$$
\chi_{\text {a.c. }}=\mathscr{J} N / \phi_{0}\left[\partial / \partial H(\partial \phi / \partial H)+1 / \phi_{0}(\partial \phi / \partial H)^{2}\right]
$$

Thus, if a magnetic flux flowing through SSL is a function of the magnetic field, ac magnetic susceptibility also depends on $H$.

By using ultrametric space representation for the glass phase of a superconductor (Ginzburg 1986), namely putting the interval between two points of time $t_{1}$ and $t_{2}$ as $Z\left(t_{1}-t_{2}\right)=\alpha \ln / t_{1}-t_{2} / \tau$ where $\tau$ is the paramagnetic time we arrive at the relaxation of the "frozen" magnetic flux according to the exponential law

$$
\phi=\phi(0) \exp [-t / \tau(H)]
$$

$t$ is the time of measurement, $\tau(H)$ is the relaxation time-dependent on the external magnetic field. For $t / \tau \ll 1$ from (9), (10) we obtain

$$
\chi_{\text {a.c. }}=\mathscr{J} n S^{2} / \phi_{0}^{2}\left[1-2 f \tau+0(1 / f \tau)^{2}\right]
$$

i.e. with increased measuring field frequency, $\chi_{\text {a.c. }}$ grows approaching the limit $\mathscr{I} n S^{2} / \phi_{0}^{2}$. It is likely that with increased temperature the dependence of $\chi_{\text {a.c. }}$ on the measuring field frequency should be smooth, since there arises a possibility of transitions between metastable states. In the SSL model under study, it is easy to obtain the difference between $\chi_{\mathrm{a} . \mathrm{c} .}^{\mathrm{ZF}}$ and $\chi_{\mathrm{a} . \mathrm{c} \text {. }}^{\mathrm{FC}}$, i.e. between ac magnetic susceptibility of a sample cooling in zero magnetic field (a wide spectrum of relaxation times) and ac magnetic susceptibility of a sample cooling in a finite magnetic field. In the last case, the spectrum of relaxation times is truncated from below $\left(\tau>\tau_{\min }\right)$ that is in line with a thermodynamical stabilization of a superconducting glassy phase.

Both in superconducting and spin glasses, as $T \rightarrow T_{g}, T \rightarrow T^{*}$, where $T^{*}$ is the ergodicity temperature (Muller et a! 1987), and some of relaxation times are very large $(\tau \gg t)$. Owing to this, even at "equilibrium" cooling, slow cooling in constant magnetic field (FC) the equilibrium state is not achieved for experimental times.

Under temperature cycling in a magnetic field in the superconducting glass, "Thermomagnetic pumping" effect is observed, i.e. a sample diamagnetic moment enhancement (Klimento et al 1987).

In the SSL model a general expression for $\chi_{\text {a.c. }}^{\mathrm{ZFC}}$ has the form:

$$
\left.\chi_{\mathrm{a} . \mathrm{c} .}^{\mathrm{ZFC}} \cong \mathscr{I} n S^{2} / \phi_{0}^{2}\left(1-\sum_{i} 1 / f_{i} \tau_{i}+H \sum_{i}\left(1 / f_{i} \tau_{i}\right) \partial \tau_{i} / \partial H\right)\right)^{2}
$$

see (Clem 1988).

Under an equilibrium cooling in the magnetic field, slowly relaxing modes remain $i>j\left(\tau_{i}<\tau_{j}\right)$ and $\chi_{\text {a.c. }}^{\mathrm{ZFC}}$. They tend to an equilibrium value (Clem 1988)

$$
\chi_{\mathrm{a} . \mathrm{c} .}^{\mathrm{ZFC}} \rightarrow \chi_{\mathrm{a} . \mathrm{c} .}^{\mathrm{rC}}=\mathscr{J} n S^{2} / \phi_{0}^{2}
$$


In the case of temperature cycling within a magnetic cooling process, modes $0<1 / f_{i} \tau_{i}<1$ will appear. We can rewrite (12) retaining the terms of the first order over $1 / f_{i} \tau_{i}$ for the ac current fixed frequency

$$
\chi_{\mathrm{a.c.} .}^{\mathrm{ZFC}} \cong \mathscr{I} n S^{2} / \phi_{0}^{2}\left(1-2 f \sum_{i} 1 / \tau_{i}+2 H / f \sum_{i} 1 / \tau_{i}^{2} \partial \tau_{i} / \partial H\right)+0(1 / f \tau)^{2}
$$

It is evident that the occurrence of small relaxation time modes under temperature cycling in a magnetic field will contribute mostly to the negative term $2 / f \sum_{i} 1 / \tau_{i}$ rather than to the third term in (14) since $\tau / H \geqslant \partial \tau / \partial H$. Such situation leads, to the increase in diamagnetic moment of a superconducting sample, i.e. to the thermomagnetic pumping effect, that is:

$$
\chi_{\text {a.c. }}^{\mathrm{FCT}} \lesssim \chi_{\text {a.c. }}^{\mathrm{FC}}
$$

where $\chi_{\text {a.c. }}^{\mathrm{FCT}}$ is the superconducting ceramic (glass) magnetic susceptibility under temperature cycling in a magnetic field, which was observed experimentally in Klimento et al (1987).

Under cooling in a constant magnetic field (FC) of a thermomagnetically treated superconductor, there remain slowly relaxing modes $1 / f_{i} \tau_{i} \ll 1$. Then $\chi_{\mathrm{a} . \mathrm{c} \text {. }}^{\mathrm{FC}}$ tends to a thermodynamical limit $\chi_{\text {a.c. }}^{\mathrm{FC}} \rightarrow \chi_{\text {a.c. }}^{\mathrm{MAX}}=\mathscr{J} n S^{2} / \phi_{0}^{2}$ depending either on measuring current frequency or external magnetic field. This behavior of equilibrium magnetization is observed in ceramic superconductors $\mathrm{La}-\mathrm{Ba}(\mathrm{Sr})_{3}-\mathrm{Cu}-\mathrm{O}$ (Muller et al 1987; Klimento et al 1987; Mazaki et al 1987) and $\mathrm{Ba}_{2-x} \mathrm{Y}_{1+x} \mathrm{Cu}_{3} \mathrm{O}_{7-y}$. Typical features of glass phase are detected in single crystals $\mathrm{YBa}_{2} \mathrm{Cu}_{3} \mathrm{O}_{7-x}$ (Fuominel et al 1988) in disordered Josephson media: composite superconductors, polysulphur-nitrides and in $\mathrm{TaSe}_{3}$ (Bastuscheek et al 1981).

It appears that one should draw closer attention to the properties of superconducting ceramics $\mathrm{BaPb}_{1-x} \mathrm{Bi}_{x} \mathrm{O}_{3}$ (Gabovich and Moiseev 1986) as they are similar in structure (distorted structure of ternary, perovskite) to metal-oxide ceramics $\mathrm{La}-\mathrm{Ba}(\mathrm{Sr})-\mathrm{Cu}-\mathrm{O}$ and $\mathrm{Y}-\mathrm{Ba}-\mathrm{Cu}-\mathrm{O}$.

\subsection{Percolation character of the superconducting transition}

Hamiltonian of the problem(I) both for the system of identical single superconducting loops (SSL) and that of superconducting filaments (Vinokur et al 1987) can be represented in the form

$$
\begin{aligned}
\hat{\mathscr{H}} & =-\sum_{\langle i j\rangle} \mathscr{J}_{i j} \cos \left(\phi_{i}-\phi_{j}-A_{i j}\right)=-\mathscr{J} \sum_{\langle i j\rangle} \cos \left(\phi_{i}-\phi_{j}-A_{i j}\right) \\
& =-\operatorname{Re} \sum_{\langle i j\rangle} S_{i}^{*} \mathscr{J}_{i j} S_{j}^{*}
\end{aligned}
$$

where $S_{i}=\exp \left(i \varphi_{i}\right), \mathscr{F}_{i j}$ is the Josephson junction integral between grains $i$ and $j$.

Hamiltonian (16) can be rewritten in the form:

$$
\hat{\mathscr{H}}=2 \mathscr{J} \sum_{\langle i j\rangle} S_{i}^{*} S_{j} P_{i} P_{j}
$$

where $\langle i j\rangle$ means the summation over the pairs of nearest neighbours. $P_{i}=P_{i}(\theta)$ and 
is found as

$$
\begin{aligned}
& P_{i}(\theta)= \begin{cases}1 & i \in \theta \\
0 & i \notin \theta\end{cases} \\
& N(\theta)=\sum_{i} P_{i}(\theta)
\end{aligned}
$$

$i \in \theta$ means that site $i$ is occupied with configuration $\theta, N$ is the general number of sites. Thus, the problem of determination of the superconducting transition characteristics reduces to the percolation problem of sites and bonds for the Ising model with Sherrington--Kirkpatrick hamiltonian (SK). It is natural that the results obtained will be similar to those of the Sherrington-Kirkpatrick model for the spin glass.

Configuration $\theta$ can be determined with probability (Harris 1974)

$$
P(\theta)=X^{N(\theta)}(1-x)^{N-N(t)}
$$

where $X$ is the concentration of superconducting couplings. With multiple assemblies $\Omega_{N}$ as $N \rightarrow \infty$ for the Ising system.

$$
\begin{aligned}
& 1 / N M\left(\Omega_{N}, H\right) \ll 1 / N \text { th }(H / k T) \sum_{j=1}^{N} \sum_{i=1}^{N} \sum_{m=0}^{N Z / 2} t^{m} C_{i j}\left(\Omega_{N}\right) \\
& C_{i j}\left(\Omega_{N}\right)=\sum_{\theta} P(\theta) C_{i j}^{m}\left(\Omega_{N}, \theta\right)
\end{aligned}
$$

where $t=\operatorname{th}(2 \mathscr{f} / k T), m$ is the system magnetization, $C_{i j}\left(\Omega_{N}, \theta\right)$ is the number of steps connecting sites $i$ and $j$ for configuration $\theta$ (Fisher 1967). For $N \rightarrow \infty$ according to Fisher (1967) we have

$$
\begin{aligned}
& \mu(H) \leqslant x \operatorname{th}\left(\begin{array}{c}
|H| \\
k T
\end{array}\right) \sum_{m} C_{m}(x t)^{m} \\
& C_{m}=\sum_{j} C_{m}\left(\tau_{i j}\right)
\end{aligned}
$$

Let $\mu=\lim _{n \rightarrow x} n^{-1} \ln C_{n}$ then at $X \mu t<1$ see (Fisher 1967)

$$
\text { th }\left[2 \mathscr{f} / k T_{\mathrm{u} A}(X)\right]=(x \mu)^{-1}
$$

where $T_{u B}$ is the maximum upper boundary temperature of the phase transition, in this case superconducting transition.

Since $2, \mathscr{f} / k T_{u B}<1$, expanding (24) yields

$$
T_{u B}=2.9 \mu X, K
$$

In the molecular field approach

$$
T_{0}(X)=2 g Z x / K
$$

Ising plane model for the maximum temperature of metal-oxide ceramic transition into superconducting state gives $T_{0}^{\text {site }} \approx 180 \mathrm{~K}$ at $X=0.59$ for sites and $T_{0}^{\text {bond }} \approx 250 \mathrm{~K}$ for bond problem. In $\mathrm{Y}-\mathrm{Ba}-\mathrm{Cu}-\mathrm{O}$ ceramics a percolation type superconducting transition is observed at temperatures of about $160-240 \mathrm{~K}$ (Chen et al 1987; Verkin et al 1987). 


\subsection{Lower and upper critical magnetic fields $\left(H_{c 1}, H_{c 2}\right)$}

A lower critical field in the SSL mode is determined as

$$
H_{c 1}=\phi_{0} / 2 S=h c / 4 e S
$$

According to estimates (Ebner and Stroud 1985) for the superconducting loop of area $S=100 \mathrm{~mm}^{2} H_{c 2}=0.1$ Oe. For a mean size of granules $0.7 \mu \mathrm{m}$ in $\mathrm{La}-\mathrm{Sr}-\mathrm{Cu}-\mathrm{O}$ $H_{c 1}=0.2$ Oe. Klimenko et al (1987) have shown that the occurrence of a diamagnetic moment in $\mathrm{La}-\mathrm{Sr}-\mathrm{Cu}-\mathrm{O}$ is probably due to a magnetic flux ejection from separate superconducting granules already taking place in a magnetic field of $H=0.52 \mathrm{Oe}$.

From nonlinearity of low-temperature magnetization isotherms (Klimenko et al 1987) in weak fields, it follows that the magnetic field penetrates first into an intergranular space $\left(H_{c 1}^{*} \leqslant 1 \mathrm{Oe}\right)$, then inside the superconducting granules $\left(H_{\mathrm{c} 1} \gtrsim 100 \mathrm{Oe}\right)$.

The calculation of upper critical field $H_{c 2}$ based on the curvature of $\mathrm{d} H_{c 2} / \mathrm{d} T$ near the transition into superconducting state is not reliably established now for either type of new high- $T_{c}$ metal-oxide ceramics. Its values varies between units and hundreds of tesla (Verkin et al 1988; Hidaka et al 1987; Bar'yakhtar et al 1987). Thus, in (Bar'yakhtar et al 1987) $B_{c 2}$ measurement up to $4.6 T \mathrm{~d} B_{c 2} / \mathrm{d} T$ taken in three points: "zero" normal state, $0.5 R_{N}, 0.8 R_{N}$. They change from $0.8 T / K$ to $3.75 T / K$, correspondingly the estimation of $B_{c 2}$, varies, from $72 T$ to $340 T$. The difference in dependence of $H_{c 2}(T)$ is probably due to a sample percolation structure. Therefore on applying the magnetic field, superconductivity is first destroyed at the expense of weak Josephson coupling, and then superconductivity is destroyed in the whole volume of sample. Thus, for Josephson couplings

$$
\mathscr{J} N=k T_{c v}=\mu_{\beta} g H_{c 2}
$$

where $T_{c v}$ is the superconducting transition temperature throughout the volume. For the infinite cluster $N=1 \quad k T_{c v}=\mu_{\beta} g H_{c 2}$. For metal-oxide superconductors $\mathrm{Y}-\mathrm{Ba}-\mathrm{Cu}-\mathrm{O}, \mathrm{H}_{c 2}(0)=70 T$, for $\mathrm{La}-\mathrm{Sr}-\mathrm{Cu}-\mathrm{O} H_{c 2}(0)=31 T$, (see Shalygin et al 1987). The maximum value of $H_{c 2}(0)$ for $\mathrm{Y}-\mathrm{Ba}-\mathrm{Cu}-\mathrm{O}$ superconductors is $200 \mathrm{~T}$.

\subsection{Almeida-Thoulence instability line}

It is evident that for weakly coupled superconducting clusters, in Ising model, Sherrington-Kirkpatric solution (Harris 1974; Fisher 1967) results in the AlmeidaThoulence nonergodicity line (Almeida and Thouless 1978)

$$
t_{c}=1-T_{c}(H) / T_{c}(0) \cong\left(3 / 4 h^{2}\right)^{1 / 3}\left[1-\left[\frac{1}{3}\left(\frac{3}{4}\right) h^{2}\right]^{1 / 3}\right]
$$

where $h=\mu g H, H$ is the external magnetic field.

By rewriting (29) in a different way

$$
h=\left(\frac{4}{3}\right)^{1 / 3} t_{c}^{3 / 2} /\left[1-\frac{1}{3}\left(\frac{3}{4}\right)^{1 / 3} h^{2 / 3}\right]
$$

for magnetic fields $0.1 \leqslant\left(\phi / \phi_{0}\right)\left(\phi_{0} / \not{J} S\right)<0.2$ we obtain

$$
h \sim 1 \cdot 17\left[1-T_{c}^{*}(H) / T_{c}(0)\right]^{3 / 2}
$$

where $T_{c}^{*}(H)$ is the nonergodicity temperature (Muller et al 1987).

Expression (31) corresponds to the ceramics $\mathrm{La}_{2} \mathrm{CuO}_{4-}: \mathrm{Ba}$ behaviour in the magnetic fields up to $0.4 \mathrm{~T}$ (Muller et al 1987). 
It is of interest to note that Ising model for an interaction within a limited radius in the molecular field approach inevitably causes frustration. In this system, order parameter $Q$ of glass phase is always smaller than the long range percolation parameter (Katner and Sompolinsky 1987). In fact, the frustration introduced into a planar $X-Y$ model with Josephson links determines the spin glass phase on a square lattice with multiple Parisi order parameter (Choi and Stroud 1987).

\section{Conclusion}

In the single superconducting loop model (SSL), for a weak Josephson interaction, characteristic features of superconducting glassy phase are determined. In particular, the difference in the behaviour of $\mathrm{dc}$ and ac magnetic susceptibility measured after cooling in zero magnetic field (ZFC) and cooling in finite magnetic field (FC) is studied. The frequency dependence of thermodynamic equilibrium susceptibility $\chi_{a \text {.c. }}^{\mathrm{FC}}$ is found. A "thermomagnetic pump" effect in a superconducting glass is explained.

For the SSL assembly in the molecular field approach, for the Ising model, a thermodynamical instability line of a granular superconductor is determined similar to that of Almeida-Thoulence in the Sherrington-Kirkpatrick theory of a spin glass.

In the SSL model for sites problem maximum values of the upper critical field $H_{c 2}$, a superconducting state transition temperature $T_{c}$ for metal-oxide ceramics $\mathrm{La}-\mathrm{Sr}-\mathrm{Cu}-\mathrm{O}$ and $\mathrm{Y}-\mathrm{Ba}-\mathrm{Cu}-\mathrm{O}$ are estimated.

\section{References}

Almeida R A and Thouless D J 1978 J. Phys. A11 983

Bar'yakhtar V G, Pan V M and Popov A G 1987 Fiz. Nizkikh. Temp. 13823

Bastuscheek C M, Buhram R A and Scott J C 1981 Phys. Rev. B24 6707

Binder K and Young A P 1986 Rev. Mod. Phys. 58801

Buhram R A, Bastuscheek C M, Scott J C and Kulik G P 1979 PAIP 207

Cai X, Joynt R and Larbalestier D G 1987 Phys. Ret. Lett. 582798

Chen J T, Wenger L E, McEwan C J and Logothetis E M 1987 Phys. Rev. Lett. 581972

Choi M Y and Stroud D 1987 Phys. Rev. B35 7109

Clem J R 1988 Physica C153 50

Ebner C and Stroud D 1985 Phys. Rev. B31 165

Fisher M E 1967 Phys. Rev. 162480

Fuominen M, Golden A M and Mecartey M L 1988 PJC HTS, Switzerland A220

Gabovich A M and Moiseev D B 1986 Usp. Fiz. Nauk 150599

Ginzburg S L 1986 JETP 90754

Harris A B 1974 J. Phys. C7 3082

Hidaka Y, Enomoto Y and Suzuki M 1987 J. Appl. Phys. 26 L726

Ishizawa Y, Fukunaga $O$ and Nozaki H 1987 Jpn. J. Appl. Phys. 26 L676

Katner J and Sompolinsky R 1987 Phys. Rev. Lett. 58164

Klimenko A G. Blinov A T, Yu I Vesnin and Straikov M A 1987 Pis'ma r JETP 46196

Mazaki M, Tanako M, Kanno R and Takeda V 1987 Jpn. J. Appl. Phys. 26 L780

Muller K A. Takashige M and Bednorz J G 1987 Phys. Rev. Lett. 581143

Orlando T P, Pelin H A and Foner S 1987 Phys. Ret. B35 5347

Sajeev J and Lubensky T C 1985 Phys. Rev. Lett. 551014

Shalygin I S, Konovalova I A, Lazarev V B and Tischenko E A 1987 Pis'ma v JETP 46152

Verkin B N, Banduryan B B and Baryl"nik A S 1987 Fiz. Nizkikh. Temp. 13771

Verkin B N, Dmitrenko N M, Dmitriev V M and Kartsovnik V M 1988 Fiz. Nizkikh. Temp. 1434

Vinokur V M. Ioffe A B. Larkin A I and Feigel'man M V 1987 JETP 93343 